\title{
Experimental probing of the interplay between ferromagnetism and localization in $(\mathrm{Ga}, \mathrm{Mn}) \mathrm{As}$
}

\author{
Maciej Sawicki ${ }^{1,2 \star}$, Daichi Chiba ${ }^{1,3}$, Anna Korbecka ${ }^{4}$, Yu Nishitani ${ }^{1}$, Jacek A. Majewski ${ }^{4}$, \\ Fumihiro Matsukura ${ }^{1,3}$, Tomasz Diet| ${ }^{2,3,4}$ and Hideo Ohno ${ }^{1,3}$
}

\begin{abstract}
The question of whether the Anderson-Mott localization enhances or reduces magnetic correlations is central to the physics of magnetic alloys ${ }^{1}$. Particularly intriguing is the case of $(\mathrm{Ga}, \mathrm{Mn})$ As and related magnetic semiconductors, for which diverging theoretical scenarios have been proposed ${ }^{2-9}$. Here, by direct magnetization measurements we demonstrate how magnetism evolves when the density of carriers mediating the spin-spin coupling is diminished by the gate electric field in metal-insulator-semiconductor structures of $(\mathrm{Ga}, \mathrm{Mn})$ As. Our findings show that the channel depletion results in a monotonic decrease of the Curie temperature, with no evidence for the maximum expected within the impurity-band models $s^{3,5,8,9}$. We find that the transition from the ferromagnetic to the paramagnetic state proceeds by means of the emergence of a superparamagnetic-like spin arrangement. This implies that carrier localization leads to a phase separation into ferromagnetic and non-magnetic regions, which we attribute to critical fluctuations in the local density of states, specific to the Anderson-Mott quantum transition.
\end{abstract}

Manipulation of magnetism by a gate electric field has been demonstrated in carrier-controlled ferromagnets by studies of the anomalous Hall effect ${ }^{10-14}$, resistance ${ }^{15,16}$ and splitting of a luminescence line ${ }^{17}$. Such studies provide information on spin polarization of itinerant carriers. To probe directly the effect of carrier localization on magnetism, we have developed superconducting quantum interference device (SQUID) magnetometry sensitive enough to quantitatively determine magnetization of ( $\mathrm{Ga}, \mathrm{Mn}) \mathrm{As}$ constituting the channel of metal-insulator-semiconductor (MIS) structures. The (Ga, Mn)As film was grown on a GaAs (001) substrate and had a thickness $t=3.5 \mathrm{~nm}$ and a nominal $\mathrm{Mn}$ cation concentration $x=7 \pm 1 \%$. For magnetic measurements, a series of $(\mathrm{Ga}, \mathrm{Mn})$ As-based parallel-plate capacitors with a gate area of about $10 \mathrm{~mm}^{2}$ and a $\mathrm{HfO}_{2}$ (dielectric constant $\kappa \cong 20$ ) gate insulator with a thickness $d$, ranging from 40 to $50 \mathrm{~nm}$ were prepared and found to sustain gate voltages $V_{\mathrm{G}}$ up to $\pm(12-15) \mathrm{V}$, or electric fields $E_{\mathrm{G}}=\left|V_{\mathrm{G}}\right| / d$ up to $3-4 \mathrm{MV} \mathrm{cm}^{-1}$ (see Fig. 1 and the Methods section for details as well as Supplementary Information, S1 for a transmission electron micrograph). Our as-grown material shows a Curie temperature $T_{\mathrm{C}}<25 \mathrm{~K}$, a typical value for very thin $(\mathrm{Ga}, \mathrm{Mn}) \mathrm{As}$ films ${ }^{18}$, and a reduced hole density, which brings the system near to the localization boundary, as illustrated for our channel in Supplementary Information, S2. The SQUID measurements are carried out along various crystallographic directions because of magnetic anisotropy. The results presented here correspond to the in-plane configuration, as we found that the magnetic moment remains in-plane even at low hole densities for which the easy axis assumes the perpendicular orientation in thick layers.

We obtain $T_{\mathrm{C}}\left(V_{\mathrm{G}}\right)$ from the temperature dependence of the spontaneous magnetic moment $m_{\mathrm{S}}(T)$ of the film during cooling the sample in zero magnetic field $(<1 \mathrm{Oe})$ under constant $V_{\mathrm{G}}$ applied at $T>T_{\mathrm{C}}$ (see Fig. 2a) and from measurements of $m_{\mathrm{S}}$ as a function of $V_{\mathrm{G}}$ at various temperatures (see Fig. 2b). Absolute values of $m_{\mathrm{S}}(T)$ are determined by a method outlined in the Supplementary Information, S3. From the results presented in Fig. 2a,b, we find that sample processing, presumably unintentional annealing, increases $T_{\mathrm{C}}$ to $34 \mathrm{~K}$ at zero gate voltage. The magnitude of $T_{\mathrm{C}}$ rises to $41 \mathrm{~K}$ under a negative bias of $V_{\mathrm{G}}=-12 \mathrm{~V}$, which increases the hole density, and can conversely be reduced to $24 \mathrm{~K}$ under a positive voltage $V_{\mathrm{G}}=+12 \mathrm{~V}$.

Within the impurity-band models of electronic states in ferromagnetic $(\mathrm{Ga}, \mathrm{Mn}) \mathrm{As}$ (refs 3, 5, 8, 9), the magnitude of $T_{\mathrm{C}}$ is expected to reach a maximum, when the Fermi level is shifted across the peak in the density of the impurity states. So far, however (Fig. 2 and refs 12,13 ), a monotonic decrease of $T_{\mathrm{C}}$ is observed when the channel is depleted by the gate voltage.

Figure 2c shows the isothermal switching among different magnetic states in the time domain, which provides the absolute scale of the magnetic moment changes reported previously for (In, Mn)As (refs 10, 12) and (Ga, Mn)As (refs 13, 14). In Fig. 2a, $m_{\text {Sat }}=m_{\mathrm{S}}(T \rightarrow 0)$ changes monotonically in the whole range of $V_{\mathrm{G}}$ (see also Fig. 2d), indicating that the channel is partially depleted at $V_{\mathrm{G}}=0$ as no increase of magnetic response is expected once all of the moments are involved in the ferromagnetic phase.

To find out to what extent one can describe the data without considering effects of localization, we calculate the hole density $p$ along the growth direction $z$ by using a Poisson solver under the Thomas-Fermi approximation (ref. 19; we have also developed a Poisson solver with Fermi-Dirac integration for highly degenerated semiconductors at low temperatures). As in ( $\mathrm{Ga}, \mathrm{Mn})$ As, the mean free path is shorter than the channel width and so the dimensional quantization is smeared out, which makes this semiclassical approach valid. We calculate $p(z)$ and then $m_{\mathrm{Sat}}\left(V_{\mathrm{G}}\right)$ and $T_{\mathrm{C}}\left(V_{\mathrm{G}}\right)$ in a self-consistent way adopting the hole density-of-states effective mass from the $6 \times 6 \mathrm{kp}$ model $^{2}$. Furthermore, as the density of the surface states and the degree of compensation are unknown, we treat the concentration of positive interface states $N_{\mathrm{i}}$ and the net concentration $N_{\mathrm{A}}$ of $\mathrm{Mn}$ acceptors in the $(\mathrm{Ga}, \mathrm{Mn})$ As channel as two adjustable parameters to obtain a $p(z)$ that describes the experimental values of $m_{\mathrm{Sat}}\left(V_{\mathrm{G}}\right) / m_{\mathrm{Sat}}(0)$.

\footnotetext{
${ }^{1}$ Laboratory for Nanoelectronics and Spintronics, Research Institute of Electrical Communication, Tohoku University, Katahira 2-1-1, Aoba-ku, Sendai 980-8577, Japan, ${ }^{2}$ Institute of Physics, Polish Academy of Sciences, al. Lotników 32/46, PL-02 668 Warszawa, Poland, ${ }^{3}$ Semiconductor Spintronics Project, Exploratory Research for Advanced Technology, Japan Science and Technology Agency, Sanban-cho 5, Chiyoda-ku, Tokyo 102-0075, Japan, ${ }^{4}$ Institute of Theoretical Physics, University of Warsaw, ul. Hoża 69, PL 00681 Warszawa, Poland. *e-mail: mikes@ifpan.edu.pl.
} 
a

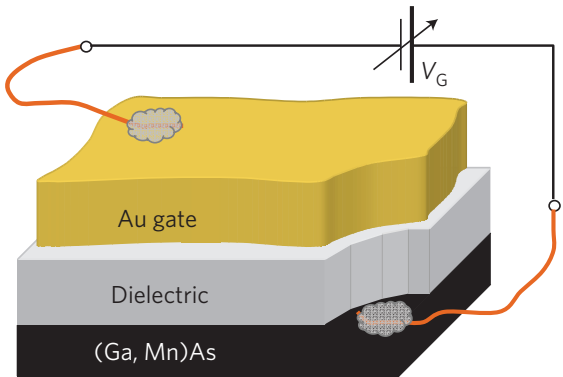

b

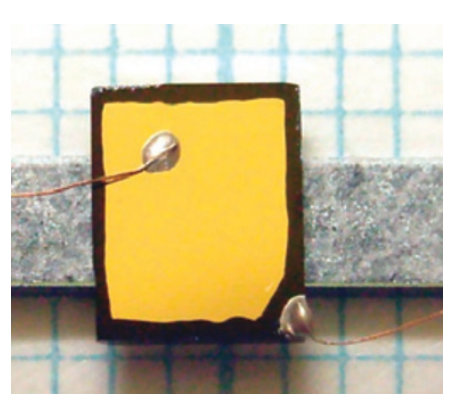

Figure 1 | General layout and an example of an investigated MIS structure. a, The arrangement of the main components of devices fabricated for our

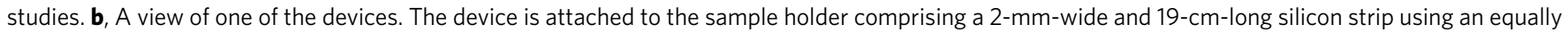
long strip of a thin double-sided low-tack sticky tape. The connections to the voltage terminals at the far ends of the sample holder are made by a high-purity $50 \mu \mathrm{m}$ copper wire.

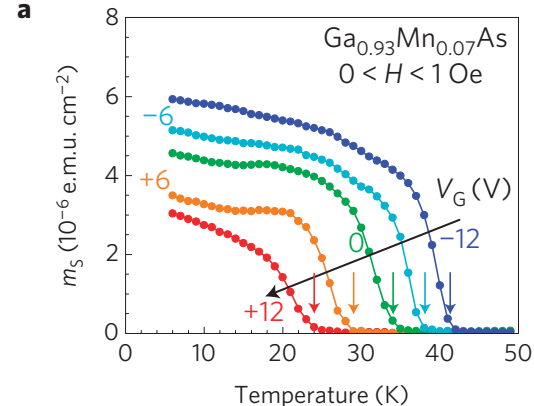

d

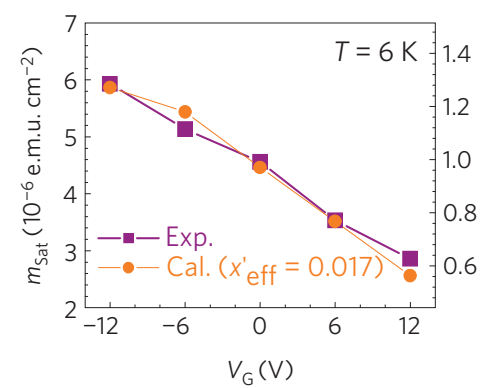

b

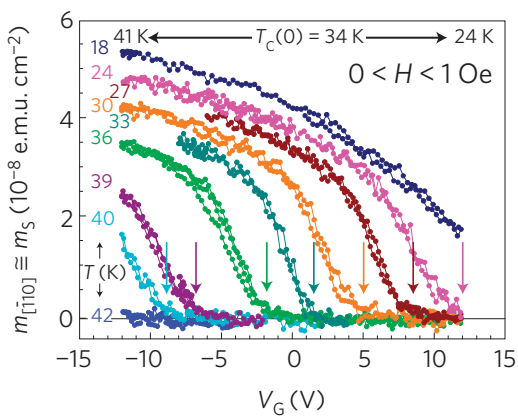

e

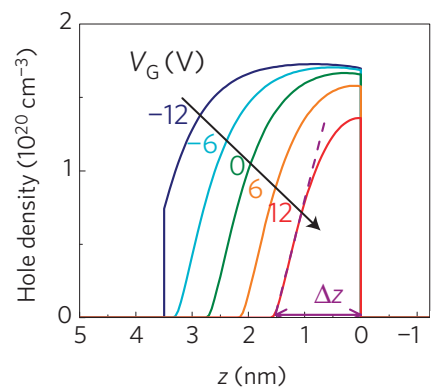

C

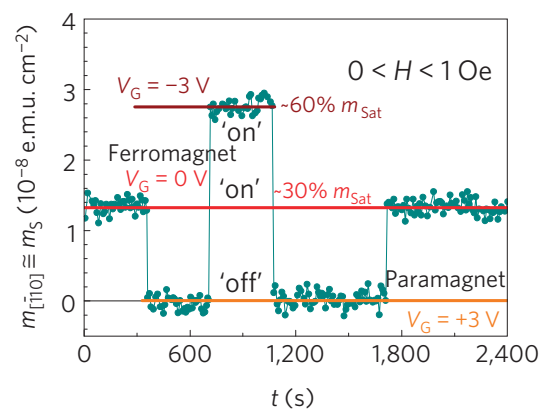

f

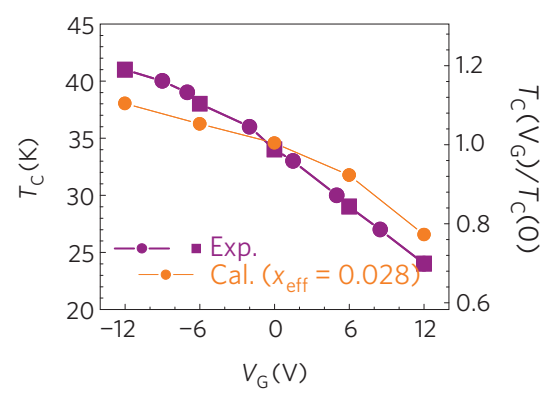

Figure 2 | Magnetic studies of the (Ga, Mn)As channel under the influence of the electric fields. a, Experimental temperature dependence of the spontaneous moment $m_{S}$ for selected values of gate voltage $V_{G}$. Temperatures at which $m_{S}$ disappears define the $C u r i e$ temperature $T_{C}$, as marked by arrows. b, Isothermal $m_{\mathrm{S}}\left(V_{\mathrm{G}}\right)$. The arrows mark the Curie points for each $T$. c, Isothermal control of $m_{\mathrm{S}}$ including complete switching 'on' and 'off' of the ferromagnetic state of the gated part of the sample at $1 \mathrm{~K}$ below $T_{C}=34 \mathrm{~K}$. The temporal resolution of the experiment is limited mainly by the acquisition

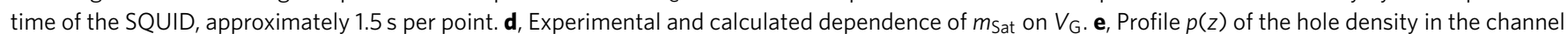
for various $V_{\mathrm{G}}$ computed assuming the interfacial donor concentration $N_{\mathrm{i}}=2.4 \times 10^{13} \mathrm{~cm}^{-2}$ and the net acceptor concentration $N_{\mathrm{A}}=2.0 \times 10^{20} \mathrm{~cm}-3$ in

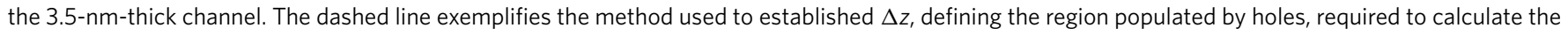
saturation magnetic moment $m_{\mathrm{Sat}}$. $\mathbf{f}$, Experimental dependence of $T_{\mathrm{C}}$ on $V_{\mathrm{G}}$ : squares (from $\mathbf{a}$ ) and circles (from $\mathbf{b}$ ). The orange circles are calculated from equation (1) assuming the hole profiles of $\mathbf{e}$.

As shown in Fig. 2d, the calculated dependence of the relative magnetic moment $m_{\mathrm{Sat}}\left(V_{\mathrm{G}}\right) / m_{\mathrm{Sat}}(0)=\Delta z\left(V_{\mathrm{G}}\right) / \Delta z(0)$, where $\Delta z\left(V_{\mathrm{G}}\right)$ is the thickness of the layer populated by the holes (see Fig. 2e), reproduces well the experimental values. This points to a strongly non-uniform hole distribution across the width of the channel for all gate voltages examined here. Furthermore, the presence of a surface depletion layer shows that an efficient steering of the hole density towards enhanced $T_{\mathrm{C}}$ or anisotropy switching at the high- $p$ end ${ }^{20}$ requires the reduction of interface states.

To calculate $T_{\mathrm{C}}\left(V_{\mathrm{G}}\right)$, we noted that the phase coherence length of holes, as extrapolated from the low-temperature data ${ }^{21}$, is expected to exceed the width of ( $\mathrm{Ga}, \mathrm{Mn})$ As channel, which implies a collective two-dimensional behaviour of the Mn spins across the channel. In this case, in terms of the sheet hole density $p_{\mathrm{s}}=\int \mathrm{d} z p(z)$ and the corresponding thermodynamic density of states at the
Fermi level $\rho_{\mathrm{s}}\left(E_{\mathrm{F}}\right)=\partial p_{\mathrm{s}} / \partial E_{\mathrm{F}}, T_{\mathrm{C}}$ according to the $p-d$ Zener model $^{2,22}$ can be written in the form,

$$
T_{\mathrm{C}}\left(V_{\mathrm{G}}\right)=\int \mathrm{d} z T_{\mathrm{C}}\left[p(z), x_{\text {eff }}\right] \int \mathrm{d} z p^{2}(z) / p_{\mathrm{s}}^{2}
$$

where $T_{\mathrm{C}}\left[p, x_{\text {eff }}\right]$ is the Curie temperature computed earlier ${ }^{2}$ for ( $\mathrm{Ga}, \mathrm{Mn}) \mathrm{As}$ as a function of the hole concentration and the effective concentration of $\mathrm{Mn}$ participating in the ferromagnetic ordering $x_{\text {eff }} N_{0}$, where $N_{0}$ is the cation concentration. As shown in Fig. 2f, on the basis of profiles from Fig. 2e, equation (1) can explain the dependence of $T_{\mathrm{C}}\left(V_{\mathrm{G}}\right) / T_{\mathrm{C}}(0)$ reasonably well. We note also that, although with the fixed values of $N_{\mathrm{i}}$ and $N_{\mathrm{A}}$ we can describe both $m_{\text {Sat }}\left(V_{\mathrm{G}}\right) / m_{\text {Sat }}(0)$ and $T_{\mathrm{C}}\left(V_{\mathrm{G}}\right) / T_{\mathrm{C}}(0)$, the fitting could be improved by taking the thickness of $(\mathrm{Ga}, \mathrm{Mn})$ As, owing 
a

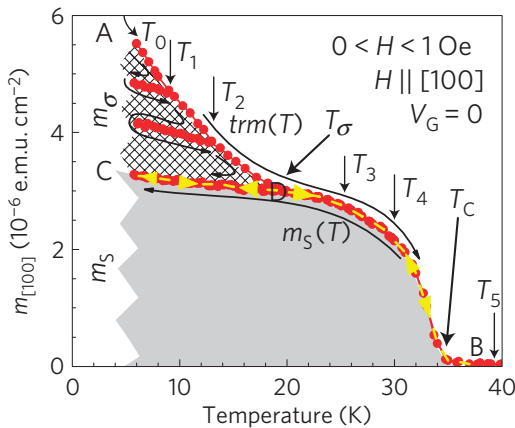

b

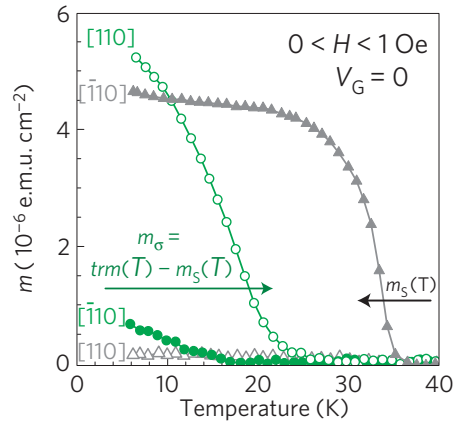

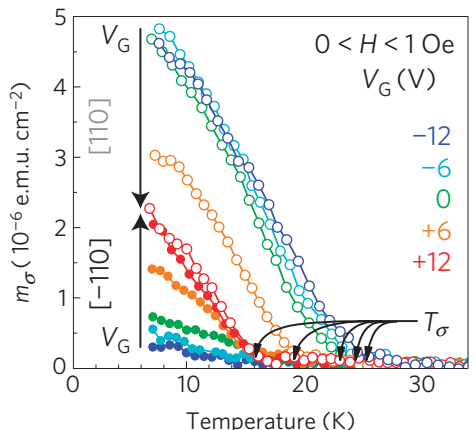

Figure 3 | Experimental evidence for the coexistence of ferromagnetic and superparamagnetic-like regions specified by differing magnetic anisotropy. a, After field cooling in $H=1 \mathrm{kOe}$, a standard measurement of thermoremanent magnetization [trm $(T)$, red points] along the [100] direction starts at the initial temperature $T_{0}=6 \mathrm{~K}$ (point $\mathrm{A}$ ) after the field quenching. Then, instead of warming the system directly to above $T_{\mathrm{C}}=34 \mathrm{~K}\left(T_{5}\right.$, point $\left.\mathrm{B}\right)$, the temperature sweep is interrupted at some intermediate temperatures ( $T_{1}$ to $\left.T_{4}\right)$ and redirected back to $T_{0}-$ where the warming is restarted to the next $T_{\mathrm{i}}$. After exceeding a characteristic temperature $T_{\sigma} \approx 19 \mathrm{~K}$ (point $\mathrm{D}$ ), all of the experimental points fall onto the same temperature-reversible line (C-D-B, dashed yellow). The procedure allows us then to identify in general: (1) the temperature-reversible component $m_{S}$ (the grey area under the C-D-B line) and (2) the history-dependent part $m_{\sigma}$ (cross hatched triangle A-D-C 'above' the C-D-B line); or in particular their projections. $\mathbf{b}$, Temperature dependence of [-110] (filled points) and [110] (open points) components of $m_{\mathrm{S}}$ (triangles) and $m_{\sigma}$ (circles), showing that the easy axis for most of $m_{\sigma}$ is [110], that is perpendicular to the easy axis of $m_{\mathbf{S}}$. c , Demonstration that $T_{\sigma}$ (black arrows) and uniaxial magnetic anisotropy of $m_{\sigma}$ (difference between open and filled points) decreases with the channel depletion, the anisotropy vanishing entirely for $V_{G}=+12 \mathrm{~V}$.

to the possible existence of a native oxide layer ${ }^{16}$, and/or the concentration of As-antisite donors in the GaAs buffer layer as extra adjustable parameters.

In ( $\mathrm{Ga}, \mathrm{Mn}) \mathrm{As}$, the self-compensation proceeds through the creation of $\mathrm{Mn}$ interstitial double donors of concentration ${ }^{23}$ $x_{\mathrm{I}} N_{0}=\left(x N_{0}-N_{\mathrm{A}}\right) / 3$. According to both theoretical ${ }^{24,25}$ and experimental $^{26}$ findings, they do not couple to the hole spins, so that $x_{\text {eff }}=x-x_{\mathrm{I}}$. Indeed, by fitting the absolute values of $T_{\mathrm{C}}\left(V_{\mathrm{G}}\right)$ and $m_{\mathrm{Sat}}\left(V_{\mathrm{G}}\right)$, we obtain reduced values of $x, x_{\text {eff }}\left(\right.$ from $\left.T_{\mathrm{C}}\right)=0.028$ and $x_{\text {eff }}$ (from $m_{\text {Sat }}$ ) $=0.017$, as shown in Fig. $2 \mathrm{f}$ and $\mathrm{d}$, respectively. According to the determined values of $N_{\mathrm{A}}=2.0 \times 10^{20} \mathrm{~cm}^{-3}, x_{\mathrm{I}}$ is 0.020 , which gives $x_{\text {eff }}=0.05$. We see that $x_{\text {eff }}$ determined from experiments is smaller than this value, particularly in the case of $m_{\mathrm{Sat}}\left(V_{\mathrm{G}}\right)$. In the search for the missing magnetic moments, we have undertaken an elaborated method of magnetization determination for this sample.

As shown in Fig. 3a, by cooling in the magnetic field we reveal the presence of an increased (with respect to $m_{\mathrm{S}}$ ) lowtemperature magnetic remanent signal. This extra contribution becomes visible only below a certain characteristic temperature $T_{\sigma}<T_{\mathrm{C}}$. Whereas the already discussed spontaneous magnetic moment $m_{\mathrm{S}}$ shows properties expected for standard ferromagnets, the properties of this extra magnetic moment $m_{\sigma}$ are similar to those shown by a collection of magnetic nanoparticles. We hereafter call it a superparamagnetic-like moment and identify $T_{\sigma}$ as a relevant blocking temperature. Interestingly, as shown in Fig. 3b, the moments $m_{\sigma}$ (circles) and $m_{\mathrm{S}}$ (triangles) are oriented in the mutually perpendicular directions, along [110] and [-110], respectively. This behaviour is typical for $(\mathrm{Ga}, \mathrm{Mn}) \mathrm{As}$, according to results of SQUID measurements for other samples collected in the Supplementary Information, S4. At the same time, a Brillouin-like character of the critical decay of magnetization at $T \rightarrow T_{\mathrm{C}}^{-}$, shown in Figs 2 and 3, and Supplementary Information, S4a,b, demonstrates the collective behaviour of the Mn spins across the channel, which confirms that the phase coherence length of holes is longer than the channel width in these samples.

To interpret these observations, we note that according to some of the present authors ${ }^{8,27}$, properties of carrier-mediated ferromagnets are strongly influenced by the vicinity of the metalto-insulator transition (MIT), particularly by the corresponding quantum critical fluctuations in the local density of states. These fluctuations break the spatial continuity of the ferromagnetic order, leading to a nanoscale phase separation, a scenario suggested also by Monte Carlo simulations ${ }^{7}$ and X-ray magnetic dichroism ${ }^{28}$ as well as providing an explanation for unexpected Coulomb blockade features ${ }^{29,30}$ in macroscopically uniform constrictions of ( $\mathrm{Ga}, \mathrm{Mn}$ )As. This process is seen by inspecting the data in Figs 2e and $3 \mathrm{c}$ for $V_{\mathrm{G}} \leq 0$, where the relative contribution $m_{\sigma}(T \rightarrow 0)$ to $m_{\mathrm{Sat}}$ increases on going towards more positive $V_{\mathrm{G}}$. On further depletion of the channel $\left(V_{\mathrm{G}}>0\right)$, a reduction in the volume of superparamagnetic regions is expected owing to a shortening of the localization length. The presence of this effect is corroborated by the low-field studies pointing to a rapid fall of $T_{\sigma}$ and progressive randomization of the easy-axis orientations, as seen in Fig. 3c.

The model presented here reaches beyond the case of very thin films. For any thickness, the depletion of the near-surface region or interface will introduce this superparamagnetic-like response in a proportion depending on the thickness of the film. Analogously, if a film of any thickness is brought to the MIT, for example, by decreasing $x_{\text {eff }}$ and, thus, $p$ and $\rho\left(E_{\mathrm{F}}\right)$, its magnetic properties will also be affected by the carrier localization. However, similarly to other thermodynamic properties, ferromagnetic characteristics do not show any critical behaviour at the MIT. Instead, within the present scenario, washing out of ferromagnetism by carrier localization proceeds by means of nanoscale phase separation, resulting in the gradual appearance of superparamagnetic-like properties. This means that in the insulating phase, ferromagnetic ordering is maintained only locally but, nevertheless, the apparent $T_{\mathrm{C}}$ is still satisfactorily described by the $p-d$ Zener model ${ }^{8}$. A further shortening of the correlation length (which corresponds to a mean localization length in this case) brings relevant blocking temperatures towards zero, so that no magnetic moments are observed without an external magnetic field at non-zero temperatures. Experimentally, a jump of the apparent $T_{\mathrm{C}}$ to zero occurs rather abruptly ${ }^{15}$. In the case of $(\mathrm{Ga}, \mathrm{Mn})$ As, where the MIT appears at relatively high values of $x_{\mathrm{eff}}$, and $\rho\left(E_{\mathrm{F}}\right)$, the reported Curie temperatures at the ferromagnetism boundary group around a common value of $T_{\mathrm{C}}^{*} \cong 15 \mathrm{~K}$, before jumping to zero. In agreement with this conjecture, lower than $\sim 15 \mathrm{~K}$ values of $T_{\mathrm{C}}^{*}$ have been reported for, for example, $(\mathrm{Zn}, \mathrm{Mn}) \mathrm{Te}$ (ref. 8) and (In, Mn)Sb (ref. 31), in which the MIT occurs at smaller values of $x_{\text {eff }}$ and $\rho\left(E_{\mathrm{F}}\right)$, respectively. We note that this reasoning does not rule out a continuous decay of $T_{\mathrm{C}}^{*}$ with $p$, it rather calls for more elaborate magnetic studies in this region of the magnetic phase diagram. 
We have developed a method to monitor experimentally and to describe theoretically changes in the magnetic moment and ordering temperature induced by an electric field in MIS structures of ferromagnets in which the spin-spin coupling is mediated by carriers. We have found that further progress in magnetization manipulations by an electric field requires a better control over interface states. Furthermore, our data demonstrate the coexistence of ferromagnetic-like, superparamagnetic-like and paramagnetic regions. We assign this phase separation to quantum critical fluctuations in the local density of hole states, specific to doped semiconductors in the vicinity of the Anderson-Mott MIT. Within this model, the portion of the spins that contribute to the longrange ferromagnetic order diminishes when electronic disorder increases, which eventually results in the loss of spontaneous magnetization. This occurs at higher values of disorder than that corresponding to the MIT (ref. 32). According to our results, magnetic anisotropy undergoes significant changes in this region. Although the distribution of magnetic ions has been assumed to be uniform, in many systems magnetic ions tend to aggregate. In such a case, the magnitude of the apparent Curie temperature is determined rather by a non-random distribution of magnetic ions ${ }^{27}$ than by the fluctuations in the local carrier density.

\section{Methods}

Sample growth and device fabrication for magnetic measurements. A 3.5-nm-thick film of $\mathrm{Ga}_{0.93} \mathrm{Mn}_{0.07} \mathrm{As}$ and $4 \mathrm{~nm}$ GaAs are grown at $195^{\circ} \mathrm{C}$ following $30 \mathrm{~nm}$ of $\mathrm{Al}_{0.8} \mathrm{Ga}_{0.2}$ As and $30 \mathrm{~nm}$ of GaAs buffers grown at $220^{\circ} \mathrm{C}$ and $560^{\circ} \mathrm{C}$, respectively, on a semi-insulating $\mathrm{GaAs}(001)$ substrate by molecular beam epitaxy (see Supplementary Information, S1 for other details and cross-sectional TEM of the structure). After placing the In contact on top of the (Ga, Mn)As channel, the as-grown material is introduced to an atomic layer deposition chamber for a high- $\kappa$ dielectric-oxide gate insulator at a substrate temperature of $130{ }^{\circ} \mathrm{C}$. Despite a relatively low deposition temperature, a $30-100 \%$ increase of $T_{\mathrm{C}}$ is always recorded after processing, indicating an increase of hole density and/or effective Mn concentration. MIS structures of the required dimension are defined by $\sim 0.5$-mm-wide stripes of photoresist put down on the surface before deposition of a $3 \mathrm{~nm} \mathrm{Cr} / 50 \mathrm{~nm} \mathrm{Au}$ gate electrode. After the lift-off, the final electrical connections to the $(\mathrm{Ga}, \mathrm{Mn})$ As channel and the gold metal electrode are attached by a silver-based conductive epoxy. We regard a device as a prospective one if during room-temperature current-voltage $(I-V)$ characteristics tests within $\pm 4 \mathrm{~V}$ $\left( \pm \sim 1 \mathrm{MV} \mathrm{cm}^{-1}\right.$ ), the capacitor shows no indication of leaking (flat $I-V$ 'curve' on the $100 \mathrm{pA}$ range indicating a leakage current below $10^{-10} \mathrm{~A} \mathrm{~cm}^{-2}$ ). Finally, typically one third of the devices survive multiple mounting on a magnetometer sample holder and low-temperature energizing up to the gate voltage of $12-15 \mathrm{~V}$ $\left(3-4 \mathrm{MV} \mathrm{cm}^{-1}\right)$. The use of indium for contacting the $(\mathrm{Ga}, \mathrm{Mn})$ As channel and some technical details of the experimental set-up limit reliable magnetometry of these devices to temperatures above $6 \mathrm{~K}$ and magnetic fields below a few kilo-oersted, respectively.

Received 1 April 2009; accepted 9 October 2009; published online 15 November 2009

\section{References}

1. Yu, U., Byczuk, K. \& Vollhardt, D. Ferromagnetism and Kondo insulator behavior in the disordered periodic Anderson model. Phys. Rev. Lett. 100, 246401 (2008).

2. Dietl, T., Ohno, H., Matsukura, F., Cibert, J. \& Ferrand, D. Zener model description of ferromagnetism in zinc-blende magnetic semiconductors. Science 287, 1019-1022 (2000).

3. Inoue, J., Nonoyama, S. \& Itoh, H. Double resonance mechanism of ferromagnetism and magnetotransport in (Ga-Mn)As. Phys. Rev. Lett. 85, 4610-4613 (2000).

4. Litvinov, V. I. \& Dugaev, V. K. Ferromagnetism in magnetically doped III-V semiconductors. Phys. Rev. Lett. 86, 5593-5596 (2001).

5. Berciu, M. \& Bhatt, R. N. Effects of disorder on ferromagnetism in diluted magnetic semiconductors. Phys. Rev. Lett. 87, 107203 (2001).

6. Kaminski, A. \& Das Sarma, S. Polaron percolation in diluted magnetic semiconductors. Phys. Rev. Lett. 88, 247202 (2002).

7. Mayr, M., Alvarez, G. \& Dagotto, E. Global versus local ferromagnetism in a model for diluted magnetic semiconductors studied with Monte Carlo techniques. Phys. Rev. B 65, 241202 (2002).

8. Bouzerar, R., Bouzerar, G. \& Ziman, T. Non-perturbative $V-J_{p d}$ model and ferromagnetism in dilute magnets. Europhys. Lett. 78, 67003 (2007).
9. Sheu, B. L. et al. Onset of ferromagnetism in low-doped $\mathrm{Ga}_{1-x} \mathrm{Mn}_{x} \mathrm{As}$. Phys. Rev. Lett. 99, 227205 (2007).

10. Ohno, H. et al. Electric-field control of ferromagnetism. Nature 408, 944-946 (2000).

11. Park, Y. D. et al. A group-IV ferromagnetic semiconductor: $\mathrm{Mn}_{x} \mathrm{Ge}_{1-x}$. Science 295, 651-654 (2002).

12. Chiba, D., Yamanouchi, M., Matsukura, F. \& Ohno, H. Electrical manipulation of magnetization reversal in a ferromagnetic semiconductor. Science 301, 943-945 (2003)

13. Chiba, D., Matsukura, F. \& Ohno, H. Electric-field control of ferromagnetism in (Ga, Mn)As. Appl. Phys. Lett. 89, 162505 (2006).

14. Endo, M., Chiba, D., Nishitani, Y., Matsukura, F. \& Ohno, H. Channel thickness dependence of the magnetic properties in ( $\mathrm{Ga}, \mathrm{Mn})$ As FET structures. J. Supercond. Nov. Magn. 20, 409-411 (2007).

15. Chiba, D. et al. Magnetization vector manipulation by electric-fields. Nature 455, 515-518 (2008).

16. Olejnik, K. et al. Enhanced annealing, high Curie temperature, and low-voltage gating in (Ga, Mn)As: a surface oxide control study. Phys. Rev. B 78, 054403 (2008).

17. Boukari, H. et al. Light and electric field control of ferromagnetism in magnetic quantum structures. Phys. Rev. Lett. 88, 207204 (2002).

18. Sørensen, B. S., Sadowski, J., Andresen, E \& Lindelof, P. E. Dependence of Curie temperature on the thickness of epitaxial (Ga, Mn)As film. Phys. Rev. B 66, 233313 (2002).

19. Birner, S. et al. Modeling of semiconductor nanostructures with nextnano ${ }^{3}$. Acta Phys. Polon. 110, 111-124 (2006).

20. Sawicki, M. et al. In-plane uniaxial anisotropy rotations in (Ga, Mn)As thin films. Phys. Rev. B 71, 121302(R) (2005).

21. Vila, L. et al. Universal conductance fluctuations in epitaxial GaMnAs ferromagnets: Dephasing by structural spin disorder. Phys. Rev. Lett. 98 , 027204 (2007).

22. Dietl, T., Cibert, J., Ferrand, D. \& Merle d'Aubigne, Y. Carrier-mediated ferromagnetic interactions in structures of magnetic semiconductors. Mat. Sci. Eng. B 63, 103-110 (1999).

23. Yu, K. M. et al. Effect of the location of $\mathrm{Mn}$ sites in ferromagnetic $\mathrm{Ga}_{1-x} \mathrm{Mn}_{x}$ As on its Curie temperature. Phys. Rev. B 65, 201303 (2002).

24. Jungwirth, T., Sinova, J., Mašek, J., Kučera, J. \& MacDonald, A. H. Theory of ferromagnetic (III, Mn)V semiconductors. Rev. Mod. Phys. 78, 809-864 (2006).

25. Blinowski, J. \& Kacman, P. Spin interactions of interstitial Mn ions in ferromagnetic GaMnAs. Phys. Rev. B 67, 121204 (2003).

26. Wang, K. Y. et al. Influence of the Mn interstitial on the magnetic and transport properties of (Ga, Mn)As. J. Appl. Phys. 95, 6512-6514 (2004).

27. Dietl, T. Origin of ferromagnetic response in diluted magnetic semiconductors and oxides. J. Phys. Condens. Matter 19, 165204 (2007).

28. Takeda, Y. et al. Nature of magnetic coupling between Mn ions in as-grown $\mathrm{Ga}_{1-x} \mathrm{Mn}_{x}$ As studied by X-ray magnetic circular dichroism. Phys. Rev. Lett. 100, 247202 (2008)

29. Wunderlich, J. et al. Coulomb blockade anisotropic magnetoresistance effect in a (Ga, Mn)As single-electron transistor. Phys. Rev. Lett. 97, 077201 (2006).

30. Schlapps, M. et al. Transport through ( $\mathrm{Ga}, \mathrm{Mn})$ As nanoislands: Coulomb-blockade and temperature dependence of the conductance. Phys. Rev. B 80, 125330 (2009).

31. Wojtowicz, T. et al. $\operatorname{In}_{1-x} \mathrm{Mn}_{x} \mathrm{Sb}$ - a narrow gap ferromagnetic semiconductor. Appl. Phys. Lett. 82, 4310-4312 (2003).

32. Matsukura, F., Ohno, H., Shen, A. \& Sugawara, Y. Transport properties and origin of ferromagnetism in (Ga, Mn)As. Phys. Rev. B 57, R2037-R2040 (1998).

\section{Acknowledgements}

We acknowledge discussion with Y. Norifusa and T. Endoh. The work at Tohoku University was supported by Grant-in-Aids from MEXT/JSPS, the GCOE program, the Research and Development for Next-Generation Information Technology Program (MEXT); M.S., A.K., J.A.M. and T.D. acknowledge financial support from EU (NANOSPIN EC: FP6-IST-015728 and FunDMS Advanced Grant of ERC); A.K. and J.A.M. acknowledge the support of the Polish Ministry of Science and High Education (project no. N2002 02632/0705).

\section{Author contributions}

Devices' fabrication: M.S., D.C., F.M.; experiments and data analysis: M.S., D.C., F.M.; theory: T.D., F.M., A.K., Y.N., J.A.M.; writing: T.D., M.S., H.O.; project planning: T.D., H.O., M.S.

\section{Additional information}

The authors declare no competing financial interests. Supplementary information accompanies this paper on www.nature.com/naturephysics. Reprints and permissions information is available online at http://npg.nature.com/reprintsandpermissions. Correspondence and requests for materials should be addressed to M.S. 